\title{
Identification of Secondary Metabolites in Flammulina velutipes by UPLC-Q-Exactive-Orbitrap MS
}

\author{
Ying Ding $\left(\mathbb{D},{ }^{1}\right.$ Sitan Chen $\mathbb{D}^{1},{ }^{1}$ Honglin Wang $\mathbb{D}^{1,2}$ Shanlei Li $\mathbb{D}^{1,2}$ Changyang Ma ${ }^{1}{ }^{1}$ \\ Jinmei Wang $\left(\mathbb{D},{ }^{1}\right.$ and Lili Cui $\mathbb{C}^{1,3}$ \\ ${ }^{1}$ National $R$ \& D Center for Edible Fungus Processing Technology, Henan University, Kaifeng 475004, Henan, China \\ ${ }^{2}$ Henan Longfeng Edible Fungi Industry Research Institute Co.,Ltd, Puyang 457300, China \\ ${ }^{3}$ Functional Food Engineering Technology Research Center, Kaifeng 475004, Henan, China \\ Correspondence should be addressed to Lili Cui; cuill@vip.henu.edu.cn
}

Received 10 June 2021; Revised 16 July 2021; Accepted 20 July 2021; Published 26 July 2021

Academic Editor: Xiao-zhi Tang

Copyright (c) 2021 Ying Ding et al. This is an open access article distributed under the Creative Commons Attribution License, which permits unrestricted use, distribution, and reproduction in any medium, provided the original work is properly cited.

Flammulina velutipes is the fourth largest edible fungus in China with high nutritional value. In this paper, ultrahigh-performance liquid chromatography tandem hybrid quadrupole-Orbitrap mass spectrometry (UPLC-Q-Exactive-Orbitrap MS) was used to identify the secondary metabolites of $F$. velutipes. The metabolites were identified by comparing the retention time, accurate molecular weight, and $\mathrm{MS}^{2}$ data with standard databases of mzVault and mzCloud (compound: 17,000 ${ }^{+}$) and BGI high-resolution accurate mass plant metabolome database (plant metabolite: $2500^{+}$). Finally, 26 secondary metabolites were preliminarily identified, including flavonoids, phenylpropanoids, organic acids, and steroids.

\section{Introduction}

Flammulina velutipes is also known as golden needle mushroom and winter mushroom with high nutritional value and medicinal value. According to "Analysis of the National Statistical Survey Results of Edible Fungi in 2019," F. velutipes is the fourth largest edible fungus in China with an output of $2.589,600$ tons in 2019. F. velutipes contains a variety of nutrients, including proteins, carbohydrates, mineral elements, vitamins, and crude fibers [1]. F. velutipes contains eighteen amino acids, including eight essential amino acids, of which lysine content is $1.09 \%$. It has been proved that lysine and its derivatives can promote children's growth and development and enhance memory. Therefore, F. velutipes is also known as "Zengzhi mushroom" $[2,3]$. It can not only be used as functional food but also has great potential in the development of medical and health products [4]. F. velutipes contains many active components, including polysaccharides, proteins, terpenoids, phenolic acids, and flavonoids [4-10]. Ishikawa et al. isolated and identified sesquiterpenoids enokipodins A-D with the cyathane skeleton from $F$. velutipes $[7,8]$. Five flavonoids were isolated and identified from $F$. velutipes by $\mathrm{Hu}$ et al. [10], named epicatechin, phillyrin, apigenin, kaempferol, and formononetin. F. velutipes has many pharmacological effects, such as antitumor [4], regulating immunity [4, 11], improving memory [5], antibacterial [8], antioxidation [12, 13], protecting the kidney [12], protecting the liver [14], neuroprotection [15], regulating intestinal flora [16], and improving constipation [17].

Ultrahigh-performance liquid chromatography tandem hybrid quadrupole-Orbitrap mass spectrometry (UPLC-Q-Exactive-Orbitrap MS) is a new type of liquid chromatography-mass spectrometry developed in recent years; it is also one of the techniques commonly used in metabolomics with the characteristics of high resolution, good quality and precision, and strong qualitative and quantitative abilities. It is used for the qualitative analysis of Chinese medicinal materials and can realize the rapid identification of various components [18]. At present, 
there are few systematic studies on the secondary metabolites of $F$. velutipes. Therefore, in this paper, the secondary metabolites of $F$. velutipes were investigated to provide a reference for research on the chemical composition of $F$. velutipes.

\section{Materials and Methods}

2.1. Materials. Fruiting bodies of $F$. velutipes were obtained from Henan Longfeng Industrial Co., Ltd. The specimens (no. 2020-09-09) were saved at the National Research and Development Center of Edible Fungi Processing Technology, Henan University.

2.2. Reagent. $\mathrm{d}_{3}$-Leucine, ${ }^{13} \mathrm{C}_{9}$-phenylalanine, $\mathrm{d}_{5}$-tryptophan, and ${ }^{13} \mathrm{C}_{3}$-progesterone were used as the internal standard. Both methanol (A454-4) and acetonitrile (A996-4) were of mass spectral grade, which were purchased from Thermo Fisher Scientific, USA. Ammonium formate (17843250G) was obtained from Honeywell Fluka, USA. Formic acid (50144-50 mL) was obtained from DIMKA, USA.

2.3. Preparation of the Sample. Dried fruiting bodies of F. velutipes were crushed by using the grinding machine. $200 \mathrm{~g}$ of $F$. velutipes powder was immersed with $50 \%$ ethanol $(2000 \mathrm{~mL})$ for 2 times at room temperature, each time for 3 days. The filtrate was lyophilized to obtain $87.2 \mathrm{~g}$ extract. The yield was $43.6 \%$. $50 \mathrm{mg}$ extract of $F$. velutipes was weighed, and then the sample was managed according to Yang et al. [19].

2.4. Chromatographic Conditions. The type of column was C18 Hypersil GOLD aQ $(100 \mathrm{~mm} * 2.1 \mathrm{~mm}, 1.9 \mu \mathrm{m})$. The mobile phases were $0.1 \%$ formic acid-water (liquid A) and $0.1 \%$ formic acid-acetonitrile (liquid B) with the elution gradient of $0-2$ min 5\% B; 2-22 min 5\%-95\% B; 22-27 min $95 \% \mathrm{~B} ; 27.1-30 \mathrm{~min} 5 \%$ B. $0.3 \mathrm{~mL} / \mathrm{min}, 40^{\circ} \mathrm{C}$, and $5 \mu \mathrm{L}$ were used as the flow rate, column temperature, and injection volume, respectively.

2.5. Mass Spectrometry Conditions. Ultraperformance liquid chromatography (Waters 2D UPLC, USA) tandem Q-Exactive high-resolution mass spectrometer (Thermo Fisher Scientific, USA) was used to separate and detect the metabolites. The mass spectrometry parameters were set according to Yang et al. [19]. In brief, 150-1500 and 70,000 were used as the mass range and MS resolution, respectively. 35,000 was used as $\mathrm{MS}^{2}$ resolution. The fragmentation energy was 20, 40, and $60 \mathrm{eV}$. Sheath gas flow rate and aux gas flow rate were 40 and 10 , respectively. Spray voltage $(|K V|)$ of the positive ion mode and negative ion mode was 3.80 and 3.20 , respectively. Ion capillary temperature and aux gas heater temperature were $320^{\circ} \mathrm{C}$ and $350^{\circ} \mathrm{C}$, respectively.

2.6. Data Analysis. BGI high-resolution accurate mass plant metabolome database (plant metabolite: $2500^{+}$), mzCloud database (compound: $17000^{+}$), and $\mathrm{mzV}$ ault database were used to identify the metabolites.

\section{Results}

3.1. Total Ion Chromatogram. The total ion current chromatogram of $F$. velutipes is shown in Figure 1.

3.2. Results of Metabolites' Identification. The metabolites of F. velutipes were analyzed by UPLC-Q-Exactive-Orbitrap MS, the structural identification of compounds in F. velutipes was based on the retention time, MS data, and $\mathrm{MS}^{2}$ data compared with the BGI high-resolution accurate mass plant metabolome database (plant metabolite: $2500^{+}$), mzCloud database (compound: $17000^{+}$), and $\mathrm{mzVault}$ database. The identified metabolites were classified into three grades (level 1, level 2, and level 3) according to the comparison results. The credibility sequence is as follows: level $1>$ level $2>$ level 3 . The detailed results are shown in Table 1. 26 compounds were preliminarily identified in $F$. velutipes, including 3 phenylpropanoids, 7 flavonoids, 1 steroid, and 15 organic acids.

3.2.1. Structural Analysis of Flavonoids. Flavonoids are easily deprotonated in the negative ion mode to produce ion $[\mathrm{M}-\mathrm{H}]^{-}$. Some flavonoids are protonated in the positive ion mode to produce ion $[\mathrm{M}+\mathrm{H}]^{+}$. Methylated flavonoids are prone to losing methyl to obtain ion $\left[\mathrm{M}-\mathrm{H}-\mathrm{CH}_{3}\right]^{-}$or $\left[\mathrm{M}+\mathrm{H}-\mathrm{CH}_{3}\right]^{+}$. Flavone O-glycoside mainly lost the sugar group by glycosidic bond fracturing. The parent nucleus of flavonoids is prone to RDA cracking and lost the CO group $[20,21]$.

Compound 1 had a weak quasi-molecular ion in the positive ion mode, the ion of $[\mathrm{M}+\mathrm{H}]^{+}$was $m / z 593$, and then it continuously lost rhamnosyl and glucosyl groups to obtain two fragment ions $m / z 447[\mathrm{M}+\mathrm{H} \text {-rhamnosyl }]^{+}$and $m / z$ $285[\mathrm{M}+\mathrm{H} \text {-rhamnosyl-glucosyl }]^{+}$. The ion $[\mathrm{M}+\mathrm{H}$-rhamnosyl-glucosyl $]^{+}$further lost methyl to obtain $\mathrm{m} / z 270$ and then further lost the CO group to produce $m / z 242[\mathrm{M}+\mathrm{H}$ rhamnosyl-glucosyl- $\left.\mathrm{CH}_{3}-\mathrm{CO}\right]^{+}$. It was speculated that compound 1 was linarin. Possible MS fragmentation pathway of linarin is shown in Figure 2. The cracking process of compound 3 is similar to that of compound 1 with fragment ions of $m / z 447[\mathrm{M}+\mathrm{H}]^{+}, m / z 285[\mathrm{M}+\mathrm{H}$-glucosyl $]^{+}, m / z 270\left[\mathrm{M}+\mathrm{H} \text {-glucosyl- } \mathrm{CH}_{3}\right]^{+}$, and $m / z 242$ $\left[\mathrm{M}+\mathrm{H} \text {-glucosyl- } \mathrm{CH}_{3}-\mathrm{CO}\right]^{+}$. It was speculated that compound 3 may be glycitin.

Compound 2 was deprotonated in the negative ion mode to produce ion $m / z 285[\mathrm{M}-\mathrm{H}]^{-}$and then underwent RDA cracking to obtain two fragment ions $m / z 133[\mathrm{M}-\mathrm{H}$ $\left.\mathrm{C}_{7} \mathrm{H}_{4} \mathrm{O}_{4}\right]^{-}$and $m / z 151\left[\mathrm{M}-\mathrm{H}-\mathrm{C}_{8} \mathrm{H}_{6} \mathrm{O}_{2}\right]^{-}$. It was speculated that compound 2 may be luteolin. Possible MS fragmentation pathway of luteolin is shown in Figure 3. The cracking process of compound 4 is similar to that of compound 2 with fragment ions of $m / z 269[\mathrm{M}-\mathrm{H}]^{-}, m / z \quad 117[\mathrm{M}-\mathrm{H}-$ $\left.\mathrm{C}_{7} \mathrm{H}_{4} \mathrm{O}_{4}\right]^{-}$, and $m / z 151\left[\mathrm{M}-\mathrm{H}-\mathrm{C}_{8} \mathrm{H}_{6} \mathrm{O}\right]^{-}$. It was speculated that compound 4 may be apigenin.

Compound 5 was protonated in the positive ion mode to produce the ion $m / z 301[\mathrm{M}+\mathrm{H}]^{+}$, then lost methyl to obtain $m / z 286$, and further lost the CO group to obtain $m / z 258$ $\left[\mathrm{M}+\mathrm{H}-\mathrm{CH}_{3}-\mathrm{CO}\right]^{+}$. It was speculated that compound 5 may be diosmetin. 


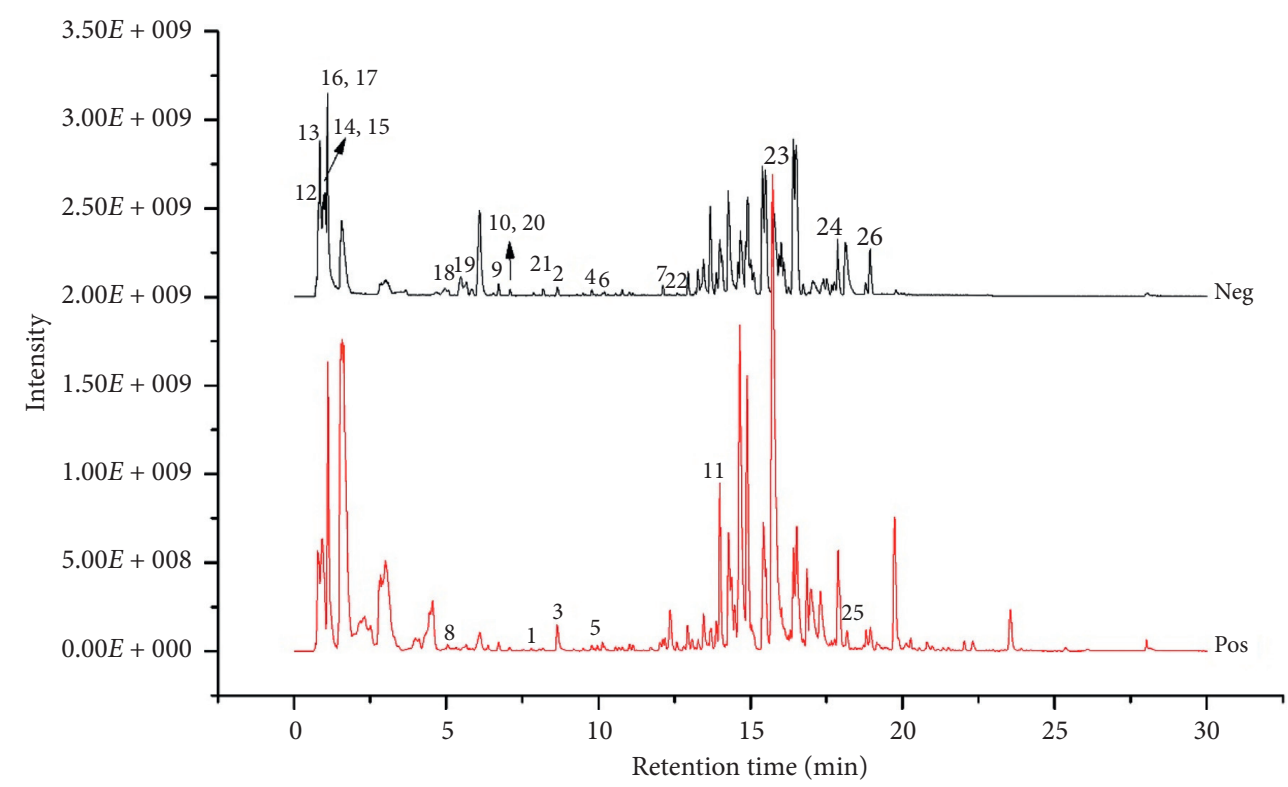

Figure 1: Total ion current chromatogram of F. velutipes in the positive ion mode (pos) and negative ion mode (neg).

Both compounds 6 and 7 contain a methoxy group, the quasi-molecular ion was $\mathrm{m} / z 299[\mathrm{M}-\mathrm{H}]^{-}$and $\mathrm{m} / z 283$ $[\mathrm{M}-\mathrm{H}]^{-}$, respectively, and then they lost the methyl unit to produce the ion $\left[\mathrm{M}-\mathrm{H}-\mathrm{CH}_{3}\right]^{-}$of $\mathrm{m} / z 284$ and $\mathrm{m} / z 268$, respectively. It was speculated that compounds 6 and 7 were hispidulin and acacetin, respectively.

\subsubsection{Structural Analysis of Phenylpropanoids.} Compound 8 was protonated in the positive ion mode to produce ion $m / z 193[\mathrm{M}+\mathrm{H}]^{+}$and then produced two fragment ions $\mathrm{m} / \mathrm{z} 165$ and $\mathrm{m} / \mathrm{z} 137$; they may be $[\mathrm{M}+\mathrm{H}-\mathrm{CO}]^{+}$and $[\mathrm{M}+\mathrm{H}-2 \mathrm{CO}]^{+}$; the cracking process of compound 8 is consistent with that of coumarins [22]. It was speculated that compound 8 may be 5,7-dihydroxy-4methylcoumarin.

Compounds 9 and 10 had the same quasi-molecular ion $\mathrm{m} / z 515[\mathrm{M}-\mathrm{H}]^{-}$, and both had characteristic fragment ions $\mathrm{m} / z 191$ [quininic acid-H] ${ }^{-}$and $\mathrm{m} / z 173$ [quininic acid-H$\left.\mathrm{H}_{2} \mathrm{O}\right]^{-}$. It was speculated that they were chlorogenic acids. The replacement position of caffeic acid can be determined according to the strength of the fragment ions [18]. Combined with the retention time, it was speculated that compounds 9 and 10 may be isochlorogenic acid B and isochlorogenic acid $\mathrm{C}$, respectively. $\mathrm{MS}^{2}$ spectrum of compounds 9 and 10 is shown in Figures 4 and 5, respectively.

3.2.3. Structural Analysis of Steroids. Compound 11 was protonated in the positive ion mode to obtain the ion $\mathrm{m} / \mathrm{z}$ $387[\mathrm{M}+\mathrm{H}]^{+}$and then continuously lost the $\mathrm{H}_{2} \mathrm{O}$ group to obtain fragment ions $\mathrm{m} / z 369\left[\mathrm{M}+\mathrm{H}-\mathrm{H}_{2} \mathrm{O}\right]^{+}$and 351 $\left[\mathrm{M}+\mathrm{H}-2 \mathrm{H}_{2} \mathrm{O}\right]^{+}[23]$; combined with the retention time and accurate molecular weight, it was speculated that compound 11 may be bufalin.
3.2.4. Structural Analysis of Organic Acids. Organic acids generally respond in the negative ion mode to produce ion $[\mathrm{M}-\mathrm{H}]^{-}$. The organic acids in F. velutipes were mostly fatty acids. They were prone to break apart and lose groups such as $\left(\mathrm{CH}_{2}\right)_{n}$ and $\mathrm{COOH}$ [24]. In this paper, organic acids in $F$. velutipes mainly produce fragments that lose $\mathrm{H}_{2} \mathrm{O}$ and $\mathrm{CO}_{2}$. The structural analysis of some organic acid compounds is as follows.

Compound 12 responded in the negative ion mode to produce ion $m / z 133[\mathrm{M}-\mathrm{H}]^{-}$, then lost the group $\mathrm{H}_{2} \mathrm{O}$ to produce ion $m / z 115\left[\mathrm{M}-\mathrm{H}-\mathrm{H}_{2} \mathrm{O}\right]^{-}$, and further lost the group $\mathrm{CO}_{2}$ to produce ion $\mathrm{m} / z$ 71[M-H-H $\left.-\mathrm{H}-\mathrm{CO}_{2}\right]^{-}$. Combined with the retention time, accurate molecular weight, and the data of [25], it was speculated that compound 12 may be DL-malic acid. The structural analysis of other organic acids is similar to that of compound 12 .

\section{Discussion and Conclusion}

Most of the compounds in F. velutipes have good biological activities. Hu et al. [15] investigated neuroprotective effects of six compounds from $F$. velutipes on $\mathrm{H}_{2} \mathrm{O}_{2}$-induced oxidative damage in PC12 cells, including arbutin, epicatechin, phillyrin, apigenin, kaempferol, and formononetin, and the results revealed that all components except apigenin mediate the apoptosis of PC12 cells via the endogenous pathway. In this paper, 7 flavonoids were identified by UPLC-Q-Exactive-Orbitrap MS, including linarin, luteolin, glycitin, apigenin, diosmetin, hispidulin, and acacetin. These flavonoids have many pharmacological effects such as antitumor, antiinflammatory, and antioxidation. Luteolin has been showing numerous therapeutic activities such as anticancer, antiinflammatory, antioxidation, and antimicrobial [26]. Apigenin has the cytostatic and cytotoxic effects on various cancer cells, prevents atherogenesis, hypertension, cardiac hypertrophy, ischemia/reperfusion-induced heart injury, 


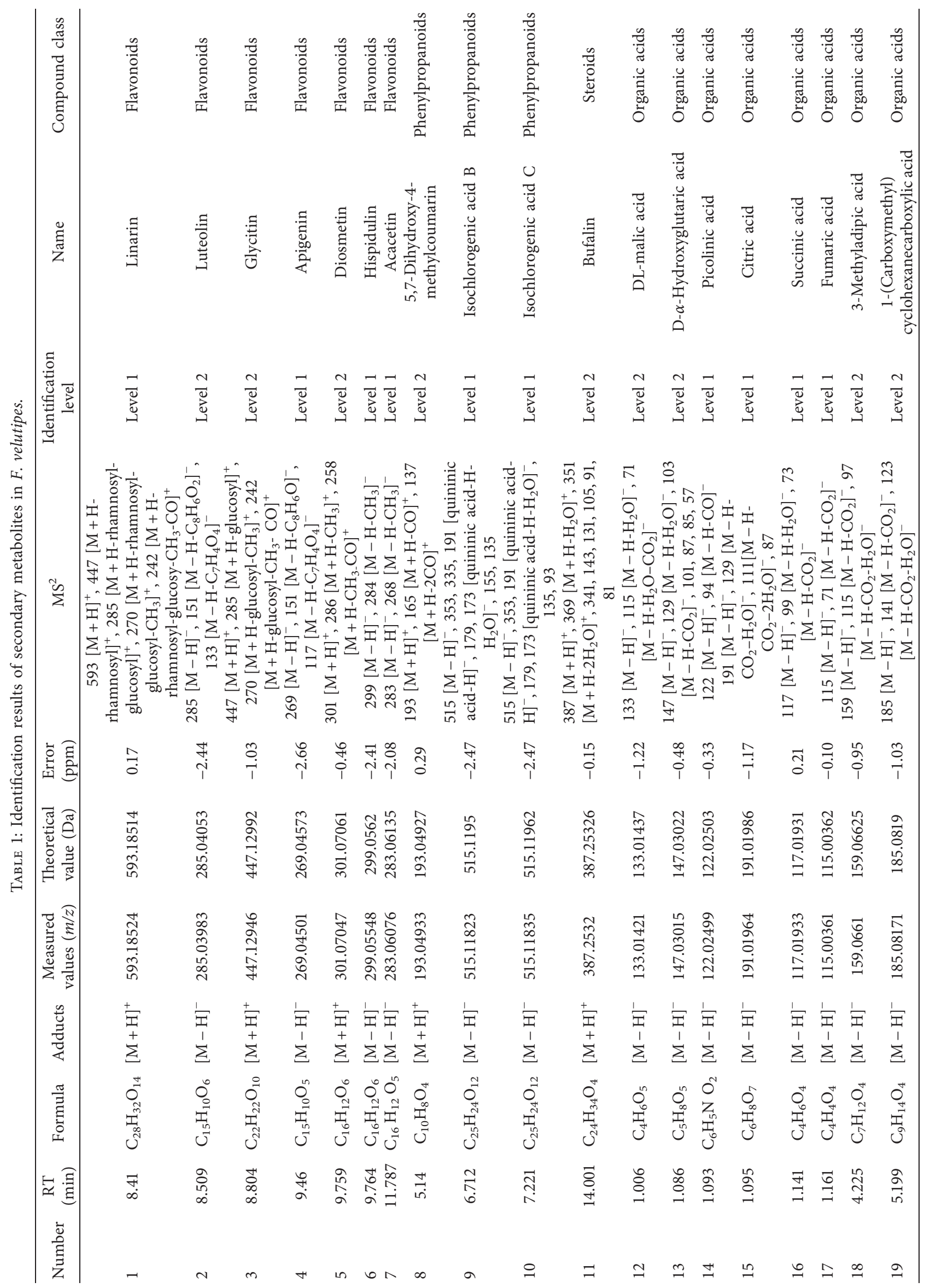




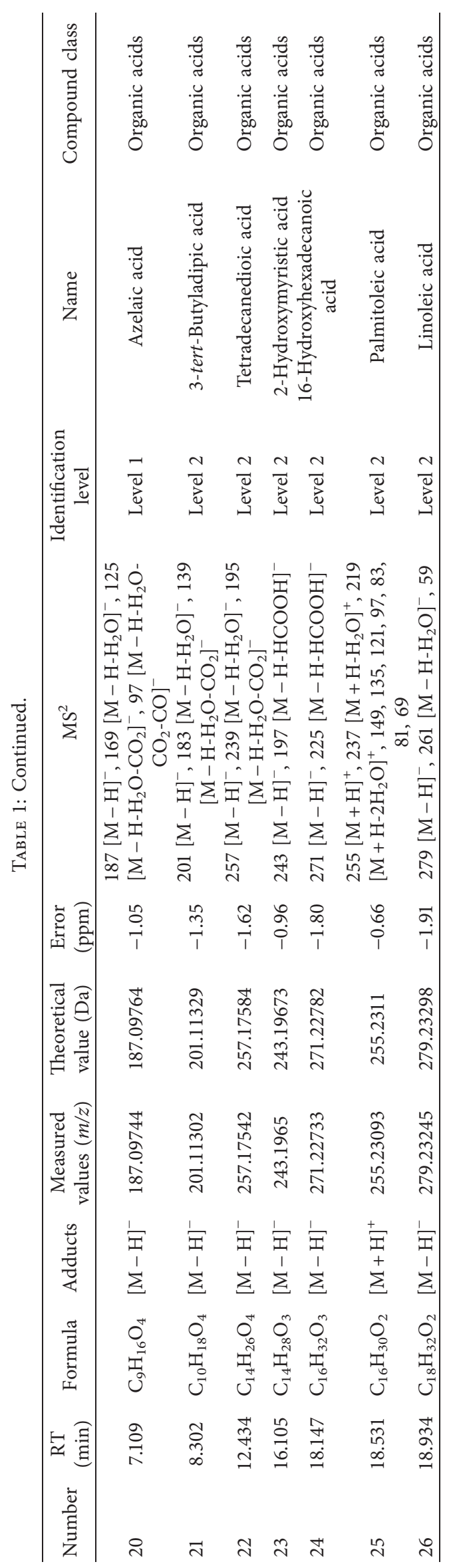


<smiles>COc1ccc(-c2cc(=O)c3ccc(O[C@@H]4O[C@H](CO[C@H]5O[C@H](C)[C@@H](O)[C@H](O)[C@H]5O)[C@@H](O)[C@H](O)[C@H]4O)cc3[o+]c2-c2ccc(OC)cc2)cc1</smiles>

FIgURe 2: Possible MS fragmentation pathway of linarin.<smiles>C#Cc1ccc(O)c(O)c1</smiles>

Figure 3: Possible MS fragmentation pathway of luteolin.

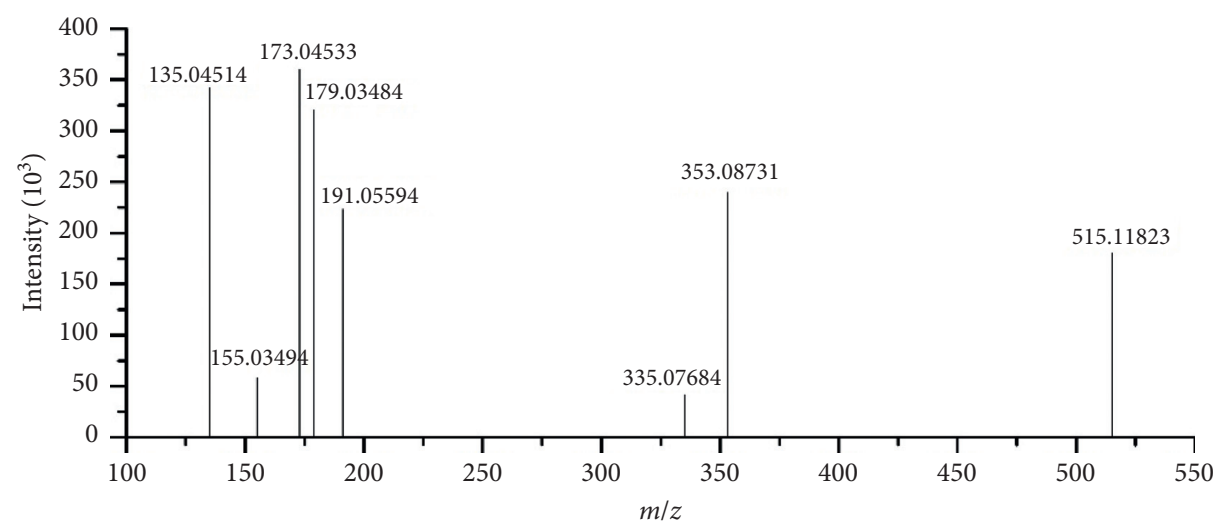

FIGURE 4: $\mathrm{MS}^{2}$ spectrum of compound 9.

and autoimmune myocarditis, protects the chemical- and ischemia/reperfusion-induced liver injury, inhibits asthma, bleomycin-induced pulmonary fibrosis, abnormal behavior, and oxygen and glucose deprivation/reperfusion-induced neural cell apoptosis, and improves pancreatitis, type 2 diabetes and its complications, osteoporosis, and collageninduced arthritis [27]. Acacetin has neuroprotective, cardioprotective, anticancer, anti-inflammatory, antidiabetic, and antimicrobial activities [28]. Hispidulin has diverse pharmacological effects such as anticancer, anti- 


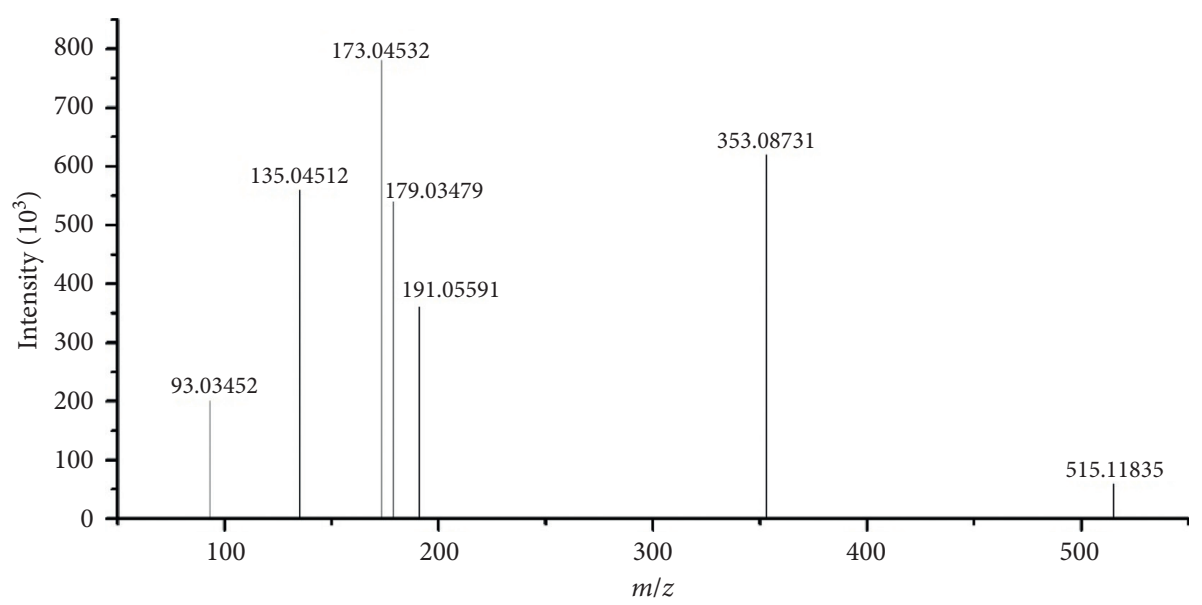

FIGURE 5: $\mathrm{MS}^{2}$ spectrum of compound 10.

inflammatory, antifungal, antiplatelet, anticonvulsant, and antiosteoporotic [29]. Linarin could suppress glioma through inhibition of $\mathrm{NF}-\kappa \mathrm{B} / \mathrm{p} 65$ and upregulating p53 expression in vitro and in vivo [30]. Glycitin has effects of alleviating lipopolysaccharide-induced acute lung injury via inhibiting NF- $\kappa \mathrm{B}$ and MAPK pathway activation in mice [31]. Diosmetin has anti-inflammatory effects on IL-4- and LPS-induced macrophage activation and the atopic dermatitis model [32]. Therefore, it is beneficial to develop flavonoids in $F$. velutipes.

One steroid (bufalin) was identified in F. velutipes in this paper. Bufalin is one of the main pharmacological and toxicological components of Venenum Bufonis and many traditional Chinese medicine preparations [33]. Currently, there is no report of bufalin in $F$. velutipes. Whether $F$. velutipes contains bufalin needs more research to determine.

Chen et al. [25] investigated chemical compositions in the stipe and pileus of F. filiformis by UPLC-Q/TOF-MS, 130 compounds were identified, including 33 amino acids and derivatives, 34 nucleotides and derivatives, 37 organic acids and lipids, 9 carbohydrate alcohols, 8 alkaloids, and 9 other compounds, and most of them were primary metabolites. Han et al. [34] investigated chemical compositions of $F$. velutipes, 11 compounds were isolated and identified, including arabinitol, ergosterol, cis-9-tricosene, uracil, nicotinamide, xanthine, glycerol, adenosine, trehalose, mannitol, and tyrosine, and most of them were primary metabolites. In this paper, 26 secondary metabolites were preliminarily identified by UPLCQ-Exactive-Orbitrap MS in F. velutipes from Henan province, including 3 phenylpropanoids, 7 flavonoids, 1 steroid, and 15 organic acids. It provides a reference for the future separation of chemical components of F. velutipes.

\section{Data Availability}

The data used to support the findings of this study are included within the article.

\section{Conflicts of Interest}

All authors declare that there are no conflicts of interest.

\section{Acknowledgments}

This work was supported by Major Public Welfare Projects in Henan Province (201300110200), Research on Precision Nutrition and Health Food, Department of Science and Technology of Henan Province (CXJD2021006), and the Key Project in Science and Technology Agency of Henan Province (212102110019 and 202102110283).

\section{References}

[1] Y. Zheng, C. Li, and N. X. Wang, "Research progress in polysaccharides from Flammulina velutipes," Food Science, vol. 31, no. 17, pp. 425-428, 2010.

[2] H. Wei, J. J. Xie, L. W. Wu et al., "Trients of the Flammulina velutipes and its functions," Natural Product Research and Development, vol. 9, no. 2, pp. 92-97, 1997.

[3] J. Y. Liu, Separation and Exploitation of Bioactive Components from Flammulina velutipes, Northwest A \& F University, Xianyang, China, 2017.

[4] H. H. Chang, K. Y. Hsieh, C. H. Yeh, Y. P Tu, and F Sheu, "Oral administration of an Enoki mushroom protein FVE activates innate and adaptive immunity and induces anti-tumor activity against murine hepatocellular carcinoma," International Immunopharmacology, vol. 10, no. 2, pp. 239-246, 2010.

[5] W. Yang, J. Yu, L. Zhao et al., "Polysaccharides from Flammulina velutipes improve scopolamine-induced impairment of learning and memory of rats," Journal of Functional Foods, vol. 18, pp. 411-422, 2015.

[6] G. E. Pelin, A. Ilgaz, K. Fatih et al., "Fatty acid compositions of six wild edible mushroom species," The Scientific World Journal, vol. 2013, Article ID 163964, 4 pages, 2013.

[7] N. K. Ishikawa, K. Yamaji, S. Tahara, Y Fukushi, and K Takahashi, "Highly oxidized cuparene-type sesquiterpenes from a mycelial culture of Flammulina velutipes," Phytochemistry, vol. 54, no. 8, pp. 777-782, 2000.

[8] N. K. Ishikawa, Y. Fukushi, K. Yamaji, S Tahara, and $\mathrm{K}$ Takahashi, "Antimicrobial cuparene-type sesquiterpenes, Enokipodins C and D, from a mycelial culture of Flammulina velutipes," Journal of Natural Products, vol. 64, no. 7, pp. 932-934, 2001.

[9] M. A. Rahman, N. Abdullah, and N. Aminudin, "Antioxidative effects and inhibition of human low density lipoprotein oxidation in vitro of polyphenolic compounds in 
Flammulina velutipes (Golden Needle Mushroom)," Oxidative Medicine and Cellular Longevity, vol. 2015, Article ID 403023, 10 pages, 2015.

[10] Q. Hu, J. Yu, W. Yang et al., "Identification of flavonoids from Flammulina velutipes and its neuroprotective effect on pheochromocytoma-12 cells," Food Chemistry, vol. 204, pp. 274-282, 2016.

[11] Z. Yin, Z. Liang, C. Li et al., "Immunomodulatory effects of polysaccharides from edible fungus: a review," Food Science and Human Wellness, vol. 10, no. 4, pp. 393-400, 2021.

[12] L. Lin, F. Cui, J. Zhang et al., "Antioxidative and renoprotective effects of residue polysaccharides from Flammulina velutipes," Carbohydrate Polymers, vol. 146, pp. 388-395, 2016.

[13] Y. Zhang, D. Wang, Y. Chen et al., "Healthy function and high valued utilization of edible fungi," Food Science and Human Wellness, vol. 10, no. 4, pp. 408-420, 2021.

[14] Y. Zhang, H. Li, T. Hu et al., "Metabonomic profiling in study hepatoprotective effect of polysaccharides from Flammulina velutipes on carbon tetrachloride-induced acute liver injury rats using GC-MS," International Journal of Biological Macromolecules, vol. 110, pp. 285-293, 2018.

[15] Q. Hu, D. Wang, J. Yu et al., "Neuroprotective effects of six components from Flammulina velutipes on $\mathrm{H}_{2} \mathrm{O}_{2}$-induced oxidative damage in PC12 cells," Journal of Functional Foods, vol. 37, pp. 586-593, 2017.

[16] J. Ye, X. Wang, K. Wang et al., "A novel polysaccharide isolated from Flammulina velutipes, characterization, macrophage immunomodulatory activities and its impact on gut microbiota in rats," Journal of Animal Physiology and Animal Nutrition, vol. 104, no. 2, pp. 735-748, 2020.

[17] X. Xin, K. W. Zheng, Y. Y. Niu et al., "Effect of Flammulina velutipes (golden needle mushroom, eno-kitake) polysaccharides on constipation," Open Chemistry, vol. 16, no. 1, pp. 155-162, 2018.

[18] B. Zeng, H. M. Liu, X. M. Liu et al., "Application of UPLC-Qexactive orbitrap MS Technology in analysis of traditional Chinese medicine," Journal of Chinese Medicinal Materials, vol. 43, no. 9, pp. 2313-2319, 2020.

[19] F. Yang, H. L. Wang, G. Q. Feng et al., "Rapid identification of chemical constituents in Hericium erinaceus based on LC-MS/ MS metabolomics," Journal of Food Quality, vol. 2021, Article ID 5560626, 10 pages, 2021.

[20] D. D. Luo, H. S. Peng, Y. Zhang et al., "Comparison of chemical components between Artemisia stolonifera and Artemisia argyi using UPLC-Q-TOF-MS," China Journal of Chinese Materia Medica, vol. 45, no. 17, pp. 4057-4064, 2020.

[21] G. H. Chang, Y. Y. Bo, J. Cui et al., "Identification on chemical constituents of aerial parts of Glycyrrhiza uralensis by UPLCQ-Exactive Orbitrap-MS," China Journal of Chinese Materia Medica, vol. 46, no. 6, pp. 1449-1459, 2021.

[22] X. Li, H. Shi, J. X. Ding et al., "Analysis of chemical constituents as flavonoids and coumarins in radix ardisiae from different sources," China Pharmacy, vol. 32, no. 4, pp. 443452, 2014.

[23] J. He, Y. Li, N. Si et al., "Comparison of the chemical composition between fresh and dried Venenum Bufonis by UPLCOrbitrap MS," Acta Pharmaceutica Sinica, vol. 49, no. 10, pp. 1446-1450, 2014.

[24] Q. L. Kang, Z. Z. Li, S. S. Fan et al., "Qualitative analysis on Perilla frutescens leaves and stalks by UPLC-Q-exactive-orbitrap-MS," Chinese Journal of Experimental Traditional Medical Formulae, vol. 26, no. 13, pp. 156-162, 2020.
[25] Y. L. Chen, Q. Li, S. J. Chen et al., "UHPLC- Q/TOF-MS Analysis on chemical compounds in stipe and pileus of Flammulina filiformis," Acta Edulis Fungi, vol. 28, no. 1, pp. 75-90, 2021.

[26] M. F. Manzoor, N. Ahmad, Z. Ahmed et al., "Novel extraction techniques and pharmaceutical activities of luteolin and its derivatives," Journal of Food Biochemistry, vol. 43, no. 9, Article ID e12974, 2019.

[27] X. Zhou, F. wang, R. J. Zhou et al., "Apigenin: a current review on its beneficial biological activities," Journal of Food Biochemistry, vol. 41, no. 4, Article ID e12376, 2017.

[28] R. B. Semwal, D. K. Semwal, S. Combrinck et al., "Acacetin-A simple flavone exhibiting diverse pharmacological activities," Phytochemistry Letters, vol. 32, pp. 56-65, 2019.

[29] K. L. Liu, F. Zhao, J. J. Yan et al., "Hispidulin: a promising flavonoid with diverse anti-cancer properties," Life Sciences, vol. 259, Article ID 118395, 2020.

[30] Z.-G. Zhen, S.-H. Ren, H.-M. Ji et al., "Linarin suppresses glioma through inhibition of NF- $\kappa \mathrm{B} / \mathrm{p} 65$ and up-regulating p53 expression in vitro and in vivo," Biomedicine \& Pharmacotherapy, vol. 95, no. 1, pp. 363-374, 2017.

[31] Y. Chen, S. Guo, K. Jiang et al., "Glycitin alleviates lipopolysaccharide-induced acute lung injury via inhibiting NF$\kappa \mathrm{B}$ and MAPKs pathway activation in mice," International Immunopharmacology, vol. 75, Article ID 105749, 2019.

[32] D. H. Lee, J. K. Park, J. Choi, H Jang, and J. W Seol, "Antiinflammatory effects of natural flavonoid diosmetin in IL-4 and LPS-induced macrophage activation and atopic dermatitis model," International Immunopharmacology, vol. 89, Article ID 107046, 2020.

[33] M. Li, X.-J. Wang, Q. Zhao et al., "Bufalin-induced cardiotoxicity: new findings into mechanisms," Chinese Journal of Natural Medicines, vol. 18, no. 7, pp. 550-560, 2020.

[34] L. Han, N. Feng, and W. Han, "Chemical components of Flammulina velutipes fruiting bodies," Acta Edulis Fungi, vol. 22, no. 4, pp. 53-57, 2015. 